\title{
A Comparison of Baseline Qinical Characteristics of Autoimmune Gastritis Patients with and without Type-1 Gastric Carcinoid Tumor
}

\author{
Onur Keskin', [MD] \\ ORCID: 0000-0002-9790-8204 \\ Mehmet Bektas ${ }^{1}$,[MD] \\ ORCID: 0000-0001-7644-4466 \\ Caglar Keskin'²,[MD] \\ ORCID: 0000-0001-5503-4468 \\ İrfan Soykan ${ }^{1},[\mathrm{MD}]$ \\ ORCID: 0000-0002-5971-4361 \\ ${ }^{1}$ Ankara University School of Medicine, Department of \\ Gastroenterology, Ankara, Turkey \\ ${ }^{2}$ Ankara University School of Medicine, Department of \\ Endocrinology, Ankara, Turkey
}

*Corresponding Author: Onur Keskin; Hacettepe Universitesi Tıp Fakültesi İç Hastalıkları Binası 2. kat; e-mail: onurkeskin@hacettepe.edu.tr;

\section{reO A B T RACT Cer}

Objectives: Autoimmune gastritis is an antibody-mediated autoimmune disease and characterized by gastric parietal cell loss. Type-1 gastric carcinoid tumor is a disease that mostly develops on the basis of autoimmune gastritis. The aim of this study is to determine similarities and differences of baseline clinical parameters between these two disorders.

Methods: Patients diagnosed as autoimmune gastritis both without gastric carcinoid tumor (n:197) and with gastric carcinoid tumor (n:40) between 2004 and 2015 at Ankara University Faculty of Medicine, Department of Gastroenterology, were included in this analysis. Data of initial signs and symptoms, basal blood count-anemia parameters, laboratory investigations, serum gastrin levels, antiparietal cell antibody status, serological helicobacter pylori markers and serum chromogranine A levels of patients were obtained, and baseline parameters of these disorders were compared Results: Patient groups were similar in age and gender. Hemoglobin, iron, total iron binding capacity, ferritin, vitamin B12 levels, antiparietal cell antibody and helicobacter pylori immunoglobulin $\mathrm{G}$ positivity rates and concomitant autoimmune thyroid disease prevalence were also similar. Median gastrin level in patients with autoimmune gastritis and gastric carcinoid tumors was significantly higher compared to the patients with autoimmune gastritis without gastric carcinoid tumors (807 vs. $1307 \mathrm{pg} / \mathrm{ml}$; p:0.006). Receiver Operating Characteristic analysis revealed that a 1000 $\mathrm{pg} / \mathrm{ml}$ threshold value for serum gastrin level is able to distinguish these two disorders with $65 \%$ sensitivity and 65\% specificity rates (area under the curve: 0.65;p:0.006). The serum chromogranine A level did not significantly differ between patient groups. Conclusion: High serum gastrin but not chromogranine A levels may be useful in deciding which patients should be followed closely in autoimmune gastritis. Keywords: gastric carcinoid tumor, autoimmune gastritis, gastrin, chromogranin A

\section{INTRODUCTION}

Autoimmune gastritis (AIG) is an organ-specific inflammatory autoimmune disorder. AIG is characterized by chronic inflammatory infiltration of gastric mucosa. In this disease, gastric parietal cell loss occurs and physiological acid secretion is impaired. The secretion of intrinsic factor also decreases with the loss of parietal cells. Decreased gastric acidity reduces the inhibition of somatostatin on gastrin secretion, and thus, the synthesis of gastrin from gastric antral cells increases [1]. Hypergastrinemia may be the result of other etiologies such as longterm use of a proton pump inhibitor (PPI) and helicobacter pylori (Hp) gastritis. There is no reported association between other etiologies of hypergastrinoma and gastric carcinoid tumor risk, but increased neuroendocrine tumor risk is well defined in AIG patients [2]. This is attributed to the trophic effect of gastrin. Chronic hypergastrinemia causes 
enterochromaffin like (ECL) cell proliferation. This proliferation may present in a wide range from mild hyperplasia of neuroendocrine glands to neuroendocrine tumors.

Type-1 gastric carcinoid tumors (Type-1 GCT) are the most common form of gastric carcinoids (70$80 \%)$ and are associated with chronic atrophic gastritis and hypergastrinemia. It usually presents with nonspecific signs and symptoms. The clinical course is usually benign, and distant metastasis is rarely seen (less than $10 \%$ of patients) [3-5]. Clinical profiles, and laboratory and endoscopic features of AIG are well known and clearly documented in previous studies [6-10].

Our aim in this analysis is to detect the similarities and differences of baseline clinical characteristics of AIG with and without type 1 GCT patients. We also investigated whether there is a parameter predicting the development of type $1 \mathrm{GCT}$ in AIG patients.

\section{MATERIALS and METHODS}

\section{Patients}

Patients diagnosed as AIG with or without type-1 GCT between 2004 and 2015 in the Ankara University gastroenterology outpatient clinic were included in the study. In this analysis, all data was obtained from the patients themselves or from medical records in the hospital computer system. The diagnosis of AIG was established on the histopathological findings in gastric biopsy tissue. Histologically, AIG is characterized by chronic inflammation and presence of gastric mucosal atrophy accompanied by loss of oxyntic glands, parietal cells and zymogen cells affecting the corpus of the stomach. Moreover, the presence of APCA and serum gastrin levels were also studied in patients. The diagnosis of type 1 GCT was based on the pathological examination of the suspected lesion, detected upon endoscopic examination.

Informed consent was obtained from all patients and the study was performed with the permission of the Ethics Committee of Ankara University Medical School.

\section{Clinical Evaluation of Patients}

Patients were evaluated according to the reason for admission to the gastroenterology outpatient clinics. The signs and symptoms of patients at the time of admission were recorded. Basically, the presenting symptoms in these patients were epigastric pain, heartburn, nausea, feeling of scratching, bloating, fullness, noise (noise coming from the abdomen), halitosis, diarrhea and weight loss. Some patients did not have any GIS complaints. These patients were referred to our clinic because of the investigation of etiology of iron deficiency anemia and vitamin B12 deficiency, and diagnosed as AIG or type-1 GCT. The main complaints in these patients were anemia-related signs and symptoms (fatigue, forgetfulness and pallor).

Also, the presence of other autoimmune diseases that can be seen together with AIG such as Graves' and Hashimoto disease, type 1 diabetes mellitus and vitiligo were investigated.

\section{Laboratory Tests and Endoscopic Studies}

Baseline complete blood count (hemoglobin, platelet count, leukocyte count, mean corpuscular volume, mean corpuscular volume of distribution), ferritin, iron, total iron binding capacity, transferrin saturation, vitamin B12, folic acid levels, erythrocyte sedimentation rate and C-reactive protein levels were recorded. Serum $\mathrm{CgA}$, gastrin levels and the presence of APCA were also detected. Serum CgA level was studied with a commercial kit (COSBIO, France; normal range: $<94 \mathrm{ng} / \mathrm{ml}$ ) and serum gastrin level was studied with the radioimmunoassay method with a commercial kit (Biosource Europe, Belgium; normal range: $28-185 \mathrm{pg} / \mathrm{ml}$ ) in our hospital laboratory. The presence of APCA was detected with an indirect immunoflourence method with the Euroimmune kit (Lübeck, Germany).

The presence of $\mathrm{Hp}$ was investigated histopathologically and serologically. HplgG was measured with the ELISA method (Trinity Biotech, Jamestown, New York, USA).

In an endoscopic examination, pallor and thinning of the corpus mucosa and visible gastric submucosal vessels are considered as signs of atrophy. In these cases, corpus and antral biopsies are taken routinely in our clinic 


\section{Statistics}

Baseline demographic characteristics, clinical features and laboratory findings of two groups were compared. The normally distributed data was analyzed with an independent sample T-test. In this analysis, mean \pm standard deviation of the data (SD) was used. Nonparametric tests (Mann-Whitney test) were performed for the analysis of the data that do not show normal distribution. The chi-square test was used for categorical variables. A correlation analysis between some parameters was also studied. In particular, we investigated the possible correlation between other parameters and CgA. Spearman's correlation test was used for this analysis.

The role of gastrin in differentiation of the patient groups was studied and threshold value was calculated for this parameter. These calculations were performed using the ROC curve. The area under the curve with $95 \%$ confidence intervals was given with sensitivity and specificity rates.

\section{RESULTS}

\section{Patient Characteristics}

The total number of patients included to this analysis was 237. Out of 237 patients, 197 patients were diagnosed as AIG without any findings of type-1 GCT, and 40 patients were diagnosed as AID and type-1 GCT histopathologically.

The age and sex of patients did not differ between groups $(52.3 \pm 13.2$ years and $136 \mathrm{~F} / 61 \mathrm{M}$ in patients with AIG; $53.3 \pm 14.7$ years and $25 \mathrm{~F} / 15 \mathrm{M}$ patients with AIG+type-1 GCT; p:0.65 and 0.26 respectively). The basic complaints at admission were abdominal-epigastric pain, flatulence (gas, bloating, etc.), nausea and/or vomiting, constipation, feeling of hunger, a feeling of fullness in the abdomen, weight loss, anorexia, lip wounds and halitosis. The main complaints of the patients that were referred to our clinic because of anemia were weakness, fatigue, forgetfulness and numbness in the hand. In some patients the diagnosis was made without any complaint. The prevalence of symptoms and signs of patients is shown in Table 1.

Table 1. Signs and symptoms of patients at baseline

\begin{tabular}{|c|c|c|c|c|}
\hline \multirow[t]{2}{*}{ Symptoms } & \multicolumn{2}{|c|}{ Autoimmune gastritis } & \multicolumn{2}{|c|}{ Type-1 Gastric Carcinoid Tumor } \\
\hline & $\mathrm{n}: 197$ & $\%$ & $\mathrm{n}: 40$ & $\%$ \\
\hline Pain & 59 & 29.9 & 12 & 30 \\
\hline Anemi symptoms & 33 & 16.7 & 6 & 15 \\
\hline Diarrhea & 19 & 9.6 & 2 & 5 \\
\hline $\begin{array}{l}\text { Reflux symptoms (regurgitation } \\
\text { and/or retrostrenal pain) }\end{array}$ & 8 & 4.0 & 0 & 0 \\
\hline Nausea and/orvomiting & 17 & 8.6 & 2 & 5 \\
\hline Gas-bloating & 58 & 29.7 & 6 & 15 \\
\hline Constipation & 9 & 4.5 & 0 & 0 \\
\hline Other & 11 & 5.5 & 0 & 0 \\
\hline No symptoms & 52 & 26.3 & 12 & 30 \\
\hline
\end{tabular}


The concomitant autoimmune diseases were investigated and autoimmune thyroid disease was present in 55 (27.9\%) of AIG and 12 (30\%) of type-1 GCT patients. Vitiligo was found in one AIG patient, and autoimmune hepatitis in one AIG patient as well. The presence of autoimmune thyroid disease was not significantly different between groups $(p=$ 0.52 ). APCA positivity was detected in $84.2 \%$ of AIG and $68.9 \%$ of type-1 GCT patients. Groups did not differ in this respect (p:0.06).

\section{Laboratory Tests}

According to baseline biochemical parameters, hemoglobin, leukocytes, platelet, serum ferritin, iron, iron binding capacity, MCV, RDW, vitamin B12, folate, MPV and CRP levels did not show significant differences in groups, as shown in Table 2 (p: 0.48; $0.62 ; 0.31 ; 0.66 ; 0.31 ; 0.32 ; 0.14 ; 0.47 ; 0.53 ; 0.7 ; 0.44$ and 0.07 , respectively).

Table 2. Baseline laboratory characteristics of groups

\begin{tabular}{|c|c|c|c|}
\hline Laboratory parameters & Autoimmune gastritis & $\begin{array}{c}\text { Type-1 gastric carcinoid } \\
\text { tumor }\end{array}$ & $\mathrm{p}$ \\
\hline Hemoglobin (g/dl) & $12.5 \pm 1.9$ & $12.7 \pm 1.6$ & 0.58 \\
\hline Leukocyte $\left({ }^{*} 10^{e} 9 / \mathrm{L}\right)$ & $6.6 \pm 1.9$ & $6.8 \pm 1.9$ & 0.48 \\
\hline Thrombocyte $\left({ }^{*} 10^{e} 9 / \mathrm{L}\right)$ & $268 \pm 76$ & $248 \pm 59$ & 0.12 \\
\hline Ferritine $(\mathrm{ng} / \mathrm{ml})$ & $19.9 \pm 36$ & $20 \pm 35$ & 0.99 \\
\hline Iron $(\mu \mathrm{g} / \mathrm{dl})$ & $58.2 \pm 37$ & $55.5 \pm 42$ & 0.71 \\
\hline Iron binding capacity $(\mu \mathrm{g} / \mathrm{dl})$ & $385 \pm 62$ & $394 \pm 78$ & 0.51 \\
\hline Vitamin B12 (pg/ml) & $215 \pm 138$ & $244 \pm 200$ & 0.28 \\
\hline Folate $(\mathrm{ng} / \mathrm{ml})$ & $9.7 \pm 3.6$ & $9.4 \pm 5.1$ & 0.7 \\
\hline Mean corpuscular volume (fL) & $82.5 \pm 10.2$ & $79.7 \pm 11.3$ & 0.21 \\
\hline Mean platelet volume (fL) & $8.73 \pm 1.2$ & $8.87 \pm 1.1$ & 0.51 \\
\hline Red cell distribution width (\%) & $15.5 \pm 2.9$ & $16.5 \pm 3.7$ & 0.14 \\
\hline C-reactive protein $(\mathrm{mg} / \mathrm{L})$ & $3.6 \pm 3.2$ & $3.9 \pm 3.5$ & 0.6 \\
\hline Serum gastrin level (pg/ml) & $\begin{array}{c}807(200-6335) \\
(n: 197)\end{array}$ & $\begin{array}{c}1307(63-3000) \\
(\mathrm{n}: 38)\end{array}$ & 0.006 \\
\hline Serum CgA (ng/ml) & $\begin{array}{c}182(46-970) \\
(\mathrm{n}: 75)\end{array}$ & $\begin{array}{c}193(25-3400) \\
(\mathrm{n}: 20)\end{array}$ & 0.77 \\
\hline
\end{tabular}

When compared groups, the percentage of patients with low levels of baseline hemoglobin ( $<12 \mathrm{~g} / \mathrm{dl})$, vitamin B12 $(<150 \mathrm{pg} / \mathrm{ml})$ and ferritin $(<25 \mathrm{ng} / \mathrm{ml})$ was not statistically different (Table 3$)$. 
Table 3. The percentage of patients with low levels of hemoglobin, ferritin and vitamin B 12 at baseline in groups

\begin{tabular}{|l|c|c|c|}
\hline & Autoimmune gastritis (n:167) & $\begin{array}{c}\text { Tip-1 gastric carcinoid } \\
\text { tumor (n:36) }\end{array}$ & $\mathrm{p}$ \\
Hemoglobin $(<12 \mathrm{gr} / \mathrm{dl})$ & $\% 42.7(80 / 187)$ & $\%(17 / 38)$ & 0.38 \\
Ferritin $(<25 \mathrm{ng} / \mathrm{ml})$ & $\% 82.4(141 / 171)$ & $\% 81.0(30 / 37)$ & 0.84 \\
Vitamin B12 $(<150 \mathrm{pg} / \mathrm{ml})$ & $\% 43.0(81 / 188)$ & $\% 31.5(12 / 38)$ & 0.14 \\
\hline
\end{tabular}

In AIG without type-1 GCT patients, the histopathological evaluation of the corpus and antrum biopsies taken during endoscopy showed Hp positivity in only three corpus and one antrum biopsy. Hp has not been detected histopathologically in any of the type-1 GCT patients.

Serum HplgG levels were measured in 149 patients. HplgG positivity was found in only 38 of 132 AIG patients (28.7\%), and 4 of 17 type-1 GCT patients (23.5\%). HplgG positivity did not show any difference between groups $(p=0.78)$.

\section{Serum Gastrin and CgA Levels}

The median gastrin level in patients with AIG and type-1 GCT was $807(200-6335) \mathrm{pg} / \mathrm{ml}$ and 1347 (396-3000) pg/ml, respectively, at baseline. The gastrin level was found to be significantly higher in the AIG+type-1 GCT patient group (p:0.006). To determine the value to be used in distinction of these two diseases, ROC analysis was performed. The threshold value of $1000 \mathrm{pg} / \mathrm{dl}$ distinguishes the two diseases with $65 \%$ sensitivity and $65 \%$ specificity. The area under curve in this analysis was determined as 0.65 [95\% confidence interval (0.55-0.75)]. ROC curve was shown in Figure 1.

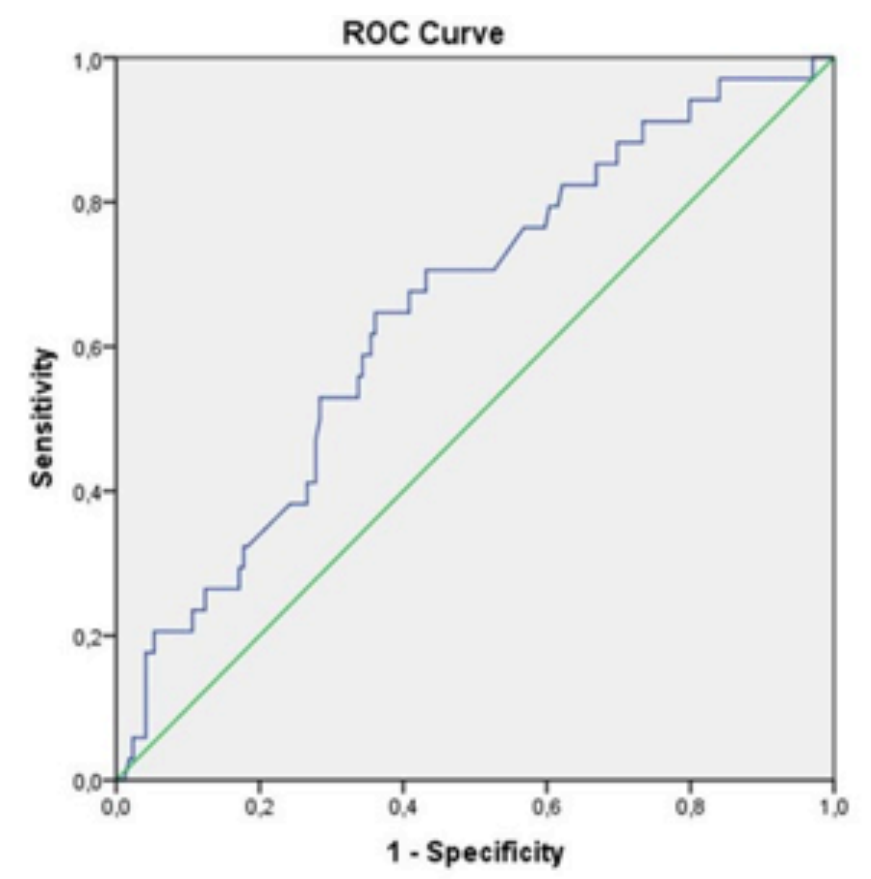

Figure 1. ROC analysis for serum gastrin levels in the differentiation of AIG patients with and without GCTs 
The CgA level could be studied in 75 AIG and 20 type-1 GCT patients. The median CgA level did not differ significantly between groups. The median CgA level in AIG and type-1 GCT patients was $182 \mathrm{ng} / \mathrm{ml}$ (46-970) and $193 \mathrm{ng} / \mathrm{ml}$ (25-3400), respectively ( $\mathrm{p}: 0.77)$.

The correlation between gastrin and $\mathrm{CgA}$ levels was also studied in the groups. In AIG without GCT patients, serum gastrin and $\mathrm{CgA}$ levels showed weak correlation (Spearman's Rho:0.29; p:0.01). When compared APCA positive to negative AIG (without type-1 GCT) patients, and the gastrin level was detected as higher in APCA positive (median gastrin:1068 vs. 742 pg/ml; p:0.02). Serum CgA level was also higher in APCA positive patients but this was not statistically significant (median CgA: 214 vs. $128 \mathrm{ng} / \mathrm{ml}$; p:0.07). In type-1 GCT patients neither serum gastrin nor serum $\mathrm{CgA}$ levels were significantly different in APCA positive and APCA negative patients [median serum gastrin 1259 vs. $1336 \mathrm{pg} / \mathrm{ml}$ (p:0.6) and median serum CgA: 310 vs. $99 \mathrm{ng} / \mathrm{ml}$ in APCA positive and negative subjects, respectively $(p: 0.1)]$.

\section{DISQUSSION}

AIG is an organ specific autoimmune disease seen in about $2 \%$, and the prevalence of it increases in Western countries [11,12]. This is characterized by loss of gastric parietal cells, and acid secretion is impaired, leading to aclorhydria and $\mathrm{G}$ cell hyperplasia. Type-1 GCT develops in a small proportion of patients in the course of AIG disease. Other situations that cause hypergastrinemia (chronic PPI usage and after vagotomy) are known not to increase the risk of gastric carcinoma development, and it has been suggested that hypergastrinemia is necessary but not sufficient for the development of type-1 GCT [3]. The clinical profiles, laboratory and endoscopic features of AIG are well known. The main purpose of our study was to reveal differences and similarities between these patients to respond to the question, "Are there any parameters that predict the development of type-1 GCT in AIG patients?"
The mean age was 53 in our study and did not differ in groups. In our study, $69 \%$ of AIG and $62.5 \%$ of type-1 GCT patients were females. This was in line with findings in other studies. There was no significant difference in gender between the AIG and type-1 GCT patients.

In our patient groups, the presenting symptoms are abdominal-epigastric pain (dyspepsia, abdominal discomfort), gas-bloating and symptoms of anemia. The prevalence of symptoms in groups is similar between the groups. In both groups, approximately $30 \%$ of patients were diagnosed incidentally without any GIS symptoms when screening tests were performed. These results indicate that presenting symptoms have no significant role in distinguishing these diseases.

Our results indicate a significant degree of iron and vitamin B12 deficiency in AIG and type-1 GCT patients. These deficiencies should be corrected, and these replacements are the important parts of the treatment. In both patient groups, there were no differences in terms of anemia parameters such as vitamin B12, hemoglobin, ferritin level and leukocyte, platelet counts. As expected, APCA positivity in both patient groups was found to be high $(84.2 \%$ vs. $68.9 \%$ ) and did not differ between groups (p:0.06). In our study, serum gastrin levels were detected as significantly higher in type-1 GCT patients when compared to AIG patients (1307 vs. $807 \mathrm{pg} / \mathrm{ml}$; $\mathrm{p}: 0.006)$ but the difference could not be observed in serum Cag A levels (193 vs. 182 ng/ml; p:0.77).

Chromogranine $A$ is widely expressed in neuroendocrine cells and, compared to healthy controls, higher levels of $\mathrm{CgA}$ levels are reported in autoimmune atrophic body gastritis, gastrinoma, multiple endocrine neoplasia syndrome type-1 patients [1316]. Although there are some studies showing the role of $\mathrm{CgA}$ in the diagnosis and prognosis of neuroendocrine tumors, data about this issue is controversial. Compana et al. showed increased CgA levels in chronic atrophic gastritis (CAG) and neuroendocrine tumors (NET) when compared to healthy controls. CgA levels did not differ significantly in CAG (especially CAG with ECL hyperplasia) and local NET disease and increasing the secretory capacity of NET 
corresponds to the increased CgA levels. [17]. Our results indicate an increased $\mathrm{CgA}$ level in AIG without GCT and AIG with type-1 GCT patients. However, the $\mathrm{CgA}$ level did not show any difference when we compare two disorders, and our results are more consistent with Compana's study. Due to our results, we may speculate that $\mathrm{CgA}$ levels increase in AIG with and without type-1 GCT, but this increase is not enough to discriminate AIG gastritis from type1 GCT.

Hypergastrinemia may be associated with different pathological states such as gastrin secreting tumors, $\mathrm{Hp}$ infection, renal failure, acid suppressing medications and chronic atrophic gastritis. In AIG related chronic atrophy, hypergastrinemia provides a proliferative and trophic effect on ECL cells, and this is strongly associated with the development of gastric carcinoid tumors. The role of gastrin in pathogenesis of gastric adenocarcinoma has also been described. Conversely, in the presence of hypergastrinemia, neuroendocrine differentiation may be observed in gastric adenocarcinoma [18-19]. Therefore, a possible pathway through neuroendocrine tumor-carcinoma has also been proposed [20]. In this context, there is a generally accepted agreement that patients with OIG and type-1 gastric carcinoid tumors should undergo regular surveillance [21]. In our study, ROC analysis for gastrin, giving the values of sensitivity and specificity of $65 \%$, considering a threshold of $1000 \mathrm{pg} / \mathrm{ml}$, shows little practical significance, gastrin level more than 1000 pg/ $\mathrm{ml}$ may be useful in deciding which patients should be under close monitoring and more careful endoscopic surveillance.

There are studies showing the role of helicobacter pylori in the development of carcinoid tumors.
Gastrin levels increase in the presence of an $\mathrm{Hp}$ infection. In an $\mathrm{Hp}$ infection, a reduction in $\mathrm{D}$ cells causes decreased somatostatin release and the disappearance of a negative effect of somatostatin on gastrin release $[22,23]$. In this group of patients, the gastrin level is reduced after $\mathrm{Hp}$ eradication. AIG patients who have an $\mathrm{Hp}$ infection histopathologically were excluded from our analysis, but patients detected HplgG positivity serologically, that shows past infection was included in the analysis. HplgG was positive in $28.7 \%$ of AIG and $23.5 \%$ of type1 GCT patients in our study and did not show any difference between groups. Clinical characteristics, laboratory parameters, serum gastrin and $\mathrm{CgA}$ levels did not show difference between HplgG positive and HplgG negative subjects.

Our study was retrospective in nature. Data of symptoms and laboratory tests could not be acquired for some patients. Chromogranin levels were not studied routinely in our clinic until 2010, and chromogranin levels could not be obtained for some patients. These are the limitations of our study.

In conclusion, AIG patients with and without type-1 GCT presented with the similar clinical and laboratory findings. The baseline serum gastrin level may be useful to differentiate these disorders with a little clinical significance, and patients who have a gastrin level more than $1000 \mathrm{pg} / \mathrm{ml}$ may undergo close surveillance, whereas serum CgA levels did not show a difference between groups.

\section{CONFUCT OF INTEREST}

The authors report no declarations of interest 
[1] Park JY, Lam-Himlin D, Vemulapalli R. Review of autoimmune metaplastic atrophic gastritis. Gastrointest Endosc. 2013;77(2):284-92.

[2] Basuroy R, Srirajaskanthan R, Prachalias A, et al. Review article: the investigation and management of gastric neuroendocrine tumours. Aliment Pharmacol Ther. 2014;39(10):1071-84.

[3] Burkitt MD, Pritchard DM. Review article: Pathogenesis and management of gastric carcinoid tumours. Aliment Pharmacol Ther. 2006;1;24(9):1305-20

[4] Rappel S, Altendorf-Hofmann A, Stolte M. Prognosis of gastric carcinoid tumours Digestion. 1995;56(6):455-62.

[5] Sato $Y$, Imamura H, Kaizaki $Y$, et al. Management and clinical outcomes of type I gastric carcinoid patients: retrospective, multicenter study in Japan. Dig Endosc. 2014;26(3):377-84

[6] Soykan I, Yakut M, Keskin O, et al. Clinical profiles, endoscopic and laboratory features and associated factors in patients with autoimmune gastritis. Digestion. 2012;86(1):20-6

[7] Tüzün A, Keskin O, Yakut M, et al. The predictive value of mean platelet volume, plateletcrit and red cell distribution width in the differentiation of autoimmune gastritis patients with and without type I gastric carcinoid tumors. Platelets. 2014;25(5):363-6.

[8] Yakut M, Keskin O, Soykan I. Effect of endogenous hypergastrinemia on gallbladder volume and ejection fraction in patients with autoimmune gastritis. Hepatobiliary Pancreat Dis Int. 2012;11(5):527-31.

[9] Soykan I, Yakut M, Keskin O. Hepatitis C virus antibody positivity may not be associated with hepatitis $C$ infection in patients with autoimmune gastritis. Eur J Gastroenterol Hepatol. 2013;25(1):122-3.

[10] Yakut M, Ustün Y, Kutlay S, et al. Multiparametric assessment of vascular function and atherosclerosis in patients with autoimmune gastritis: a comparative study. Dig Dis Sci. 2011;56(12):3583-9.

[11] Carmel R. Prevalence of undiagnosed pernicious anemia in the elderly. Arch Intern Med 1996;156:1097-1100

[12] Song $H$, Held $M$, Sandin $S$, et al. Increase in the prevalence of atrophic gastritis among adults age 35 to 44 years old in Northern Sweden between1990 and 2009. Clin Gastroenterol Hepatol 2015;13(9):1592-1600
[13] Peracchi M, Gebbia C, Basilisco G, et al. Plasma chromogranin $A$ in patients with autoimmune chronic atrophic gastritis, enterochromaffin-like cell lesions and gastric carcinoids. Eur J Endocrinol. 2005;152(3):443-8.

[14] Bashir S, Gibril F, Ojeaburu JV, et al. Prospective study of the ability of histamine, serotonin or serum chromogranin A levels to identify gastric carcinoids in patients with gastrinomas. Aliment Pharmacol Ther. 2002;16(7):1367-82.

[15] Borch K, Stridsberg M, Burman P, et al. Basal chromogranin $A$ and gastrin concentrations in circulation correlate to endocrine cell proliferation in type-A gastritis. Scand J Gastroenterol. 1997;32(3):198-202.

[16] Vannella L, Sbrozzi-Vanni A, Lahner E, et al. Development of type 1 gastric carcinoid in patients with chronic atrophic gastritis. Aliment Pharmacol Ther 2011;33(12):1361-1369

[17] Campana D, Nori F, Piscitelli L, et al. Chromogranin A: is it a useful marker of neuroendocrine tumors? J Clin Oncol. 2007;20;25(15):1967-73.

[18] [Burkitt MD, Varro A, Pritchard DM. Importance of gastrin in the pathogenesis and treatment of gastric tumors. World J Gastroenterol 2009;15(1):1-16

[19] Ovigstad G, Ovigstad T, Westre B, et al. Neuroendocrine differentiation in gastric adenocarcinomas associated with severe hypergastrinoma and/or pernicious anemia APMIS 2002;110(2):132-9

[20] Waldum HL, Ovigstad G, Falkmer S. Indications for a neuroendocrine tumor-carcinoma sequence. Virchows Arch 2001;439(2):215-7

[21] Rusznievski P, Delle Fave G, Cadiot G, et al; Frascati Consensus Conference; European neuroendoscrine Tumor Society. Well-differentiated gastric tumors/carcinomas. Neuroendocrinology 2006;84(3):158-64

[22] Sato Y, Iwafuchi M, Ueki J, et al. Gastric carcinoid tumors without autoimmune gastritis in Japan: a relationship with Helicobacter pylori infection. Dig Dis Sci 2002;47(3):579-585

[23] Kidd M, Miu K, Tang LH, et al. Helicobacter pylori lipopolysaccharide stimulates histamine release and DNA synthesis in rat enterochromaffin-like cells. Gastroenterology 1997;113(4):1110-1117 